\title{
Educar entreteniendo: los problemas de salud de los personajes femeninos en la ficción TV española*
}

\author{
Educating while Entertaining: Health Problems of Female Characters in the Spanish TV Fiction \\ Educar entretendo: os problemas de saúde das personagens femininas na ficção TV espanhola
}

\section{Charo Lacalle ${ }^{\mathrm{a}}$}

Universidad Autónoma de Barcelona, España rosario.lacalle@uab.es

ORCID: http://orcid.org/0000-0002-0024-6591

Beatriz Gómez Morales

Universidad Internacional de la Rioja, España

ORCID: http://orcid.org/0000-0002-0557-528X
DOI: https://doi.org/10.11144/Javeriana.syp37-73.eeps Redalyc: http://www.redalyc.org/articulo.oa $: \mathrm{id}=86058735006$

Fecha de recepción: 24 Marzo 2017

Fecha de aprobación: 16 Abril 2018

Fecha de publicación: 30 Diciembre 2018

\section{Resumen:}

El uso del entretenimiento en la comunicación mediática sobre salud viene siendo una estrategia habitual desde la década de los noventa; pero, aunque su eficacia ha sido reiteradamente probada, algunos estudiosos critican el sesgo de los mensajes y discuten sus beneficios. Esta investigación propone una metodología original que combina técnicas cuantitativas (SPSS) y aproximaciones cualitativas (socio-semiótica y script theory) para analizar la representación de la enfermedad, la discapacidad y el consumo de substancias tóxicas de los personajes femeninos de la ficción televisiva española. El análisis revela la ambivalencia entre la vocación didáctica de los relatos y la recurrencia de tópicos. Los resultados también muestran la escasa relevancia otorgada a la salud femenina ya la promoción de hábitos saludables.

Palabras clave: televisión, ficción, educación, entretenimiento, género.

\section{Abstract:}

The use of the entertainment in the media communication about health issues has become a usual strategy since the 1990 s. Notwithstanding, despite its effectiveness has been repeatedly proven, some scholars criticize the bias in the messages and question the benefits. This research proposes an original methodology combining quantitative techniques (SPSS) and qualitative approaches (social semiotic and script theory) to analyze the representations of the disease, disability and toxic substance use by the female characters in the Spanish TV fiction. The analysis shows the ambivalence between the teaching vocation of the stories and the recurrence of topics. Results also show the low relevance that female health and the healthy habit promotion have.

Keywords: television, fiction, education, entertainment, gender.

\section{Resumo:}

O uso do entretenimento na comunicação midiática sobre saúde vem sendo uma estratégia habitual desde a década de noventa, mas, embora a sua eficácia já tivesse sido reiteradamente comprovada, alguns estudiosos criticam o viés das mensagens e discutem os benefícios. Esta pesquisa propõe uma metodologia original que mistura técnicas quantitativas (SPSS) e aproximações qualitativas (sócio-semiótica e script theory) para analisar a representação da doença, invalidez e consumo de substancias tóxicas das personagens femininas da ficção televisiva espanhola. A análise revela a ambivalência entre a vocação didática das histórias e a recorrência de tópicos. Os resultados mesmo mostram a escassa relevância dada à saúde feminina e a promoção de hábitos saudáveis. Palavras-chave: televisão, ficção, educação, entretenimento, gênero.

Notas de autor: 


\section{Introducción}

El uso del entretenimiento para diseminar mensajes sobre salud a través de los medios se convirtió en una estrategia habitual en la década de los noventa (Glick et al., 1998; Bouman, 2014), estimulada por los avances en el control de las enfermedades infecciosas y el impacto de las dolencias crónicas y degenerativas. Los estudios sobre el rol de los medios en la educación reconocen de manera generalizada su influencia, aunque algunos investigadores contraponen el desinterés generalizado por la vertiente político social de los discursos sobre salud al interés por las historias sobre la atención médica (Hayes et al., 2007). Otros autores critican el sesgo de los mensajes y discuten sus beneficios (Lantz y Booth, 1998; Powers y Andsager, 1999). Un tercer grupo alerta sobre la creciente intimidad entre los medios y la medicina en una sociedad neocapitalista y consumidora de terapias (Hooker y Pols, 2006), y cuestiona el papel creciente atribuido a los individuos en el cuidado de su propia salud (Razum, Weishaar y Schaeffer, 2016).

Este artículo resume los resultados más relevantes de una investigación sobre la representación de los problemas relacionados con la salud (enfermedad, discapacidad y adición) de los personajes femeninos de la ficción televisiva española. El análisis realizado combina técnicas cuantitativas (SPSS) y aproximaciones cualitativas (socio-semiótica y script theory) para analizar todos los formatos de ficción doméstica de estreno emitidos a lo largo de 2012 y 2013 (series, seriales, miniseries, TV movies y sketches). La investigación parte de las siguientes premisas:

1. Los medios resultan muy eficaces para afrontar problemas de carácter social y sanitario (Wilkin et al., 2007).

2. A pesar de la expansión de la Health Education en Internet, la televisión sigue teniendo un papel preponderante en este ámbito (Payton, Kvasny y Kiwanuka-Tondo, 2014).

3. Los formatos narrativos tienen mayor capacidad persuasiva que el resto de los programas televisivos (Murphy, Frank, Chatterjee y Baezconde-Garbanati, 2013).

\section{Marco teórico}

Singhal y Rogers (1999) denominan Education Entertainment (EE) al proceso "of purposely designing and implementing a media message both to entertain and educate" (p. 9), y remontan su descubrimiento a la telenovela Simplemente Maria (Perú, Panamericana TV, 1969-1971). El desarrollo de la EE está ligado a la comunicación para la salud de los "países pobres" (Mazziotti, 2006, p. 76), y su interés reside en la "fundamentación teórico-metodológica y la aproximación práctica de la educación, el desarrollo y el cambio social" que lleva a cabo (Tufte, 2004, p. 39). Desde la perspectiva de la recepción, el EE funciona como un proceso de "resignificación educativa" por parte de la audiencia (Fuenzalida, 1996, 2011).

Los mensajes sobre la salud pueden adoptar una gran pluralidad de fórmulas (Kaiser Family Foundation, 2004; Singhal y Rogers, 2004), siempre que se integren adecuadamente en los programas y los mejoren (Glick et al., 1998). La identificación con los personajes constituye una estrategia clave en los mensajes de la EE (Igartúa y Casanova, 2016), aunque sólo suelen resultar eficaces si el destinatario se reconoce emocionalmente en dichos personajes (Fuenzalida, 1992). Algunos autores subrayan la relación directamente proporcional entre el grado de protagonismo y los efectos de la EE (Bouman, Maas y Kok, 1998), mientras que otros consideran que las storylines secundarias también pueden llegar a influenciar a los espectadores (Valente et al., 2007). Parikh (2007), Pintor-Holguín et al. (2012) y Weaver, Wilson y Langendyk (2014) constatan la influencia en los espectadores de los dramas médicos, mientras que Bury (2001) y Makoul y Peer (2004) advierten que la mezcla de realidad y ficción puede inducir imágenes distorsionadas sobre la relación médicopaciente, y apuestan por la educación crítica. 


\section{Los problemas de salud en la ficción televisiva}

Los estudios sobre los problemas de salud en la ficción televisiva, en los ámbitos representados en la ficción española, se pueden dividir, a grandes rasgos, en tres grupos: el primero está dedicado a las adiciones y cuenta con un mayor número de aportaciones que el resto; los trabajos del segundo grupo se centran la discapacidad (física y psíquica); y los del tercero, en la enfermedad.

La investigación sobre el consumo de alcohol presenta dos líneas claramente diferenciadas. Los trabajos del primer período, realizados entre los años setenta y noventa, evidencian la representación normalizada, e incluso positiva de dicho consumo; mientras que las aportaciones de la segunda etapa constatan la evolución de las representaciones e inciden en la valoración negativa que llevan a cabo. McEwen y Hanneman (1974), Hanneman y McEwen (1976), Breed y De Foe (1981), Breed, De Foe y Wallack (1984) y Hundley (1995) muestran la relación del alcohol con el ocio y con algunos valores sociales, como la amistad y la camaradería. Greenberg (1981) y Hansen (1986) observan que el consumo de alcohol es tratado como una actividad habitual y normalizada, mientras otros estudiosos revelan su asociación con personajes principalmente positivos (Breed y De Foe, 1981), y señalan que las consecuencias negativas de su ingesta tienden a ser obviadas (Furnham, Ingle, Gunter y McClelland, 1997; Parsons, Rissel y Douglas, 1999), o bien son abordadas desde el humor (Christensen, Henriksen y Roberts, 2000). Las investigaciones sucesivas evidencian, por el contrario, la prevalencia de los mensajes negativos sobre el alcohol, más en sintonía con la vertiente pedagógica de una buena parte de la televisión actual (Singhal, Cody, Rogers y Sabido, 2004; Russell y Russell, 2009). Mazziotti (2006) constata que la telenovela presenta el alcoholismo como un vicio, asociado frecuentemente con los villanos. Los estudios publicados entre finales de los años 90 y comienzos del 2000 también incluyen un tercer grupo de investigaciones que detectan la convivencia de mensajes positivos y negativos en un mismo programa (Russell, Russell y Grube, 2009; Chang y Roth, 2000; Mathios, Avery, Bisogni y Shanahan, 1998; Van Hoof, De Jong, Fennis y Gosselt, 2009).

La literatura sobre el consumo de tabaco en la ficción televisiva también es extensa. La mayor parte de las investigaciones analizan la frecuencia de las representaciones relacionadas con esta adicción, con el fin de determinar su evolución. Cruz y Wallack (1986) observan un aumento sostenido hasta mitad de los años ochenta, revalidado sucesivamente en los trabajos de Hazan y Glantz (1995), Sone (1999) y McGee y Ketchel (2006). Tanto los estudios cualitativos (Verma, Adams y White, 2007; Hanewinkel y Wiborg, 2008; Eslick y Eslick, 2009) como los cuantitativos (Cullen et al., 2011; Lyons, McNeill y Britton, 2013) constatan la presencia frecuente del hábito de fumar entre los personajes. Sone (1999), Kanda et al. (2006) y Cruz y Wallack (1986) señalan que la representación de fumadores es más frecuente en el drama que la comedia. Cumberbatch y Gauntlett (2005) y McGee y Ketchel (2006) observan que este tipo de representaciones tiende a eludir sus consecuencias negativas. Por el contrario, estos mismos autores, al igual que Christensen et al. (2000), constatan que los mensajes sobre las drogas ilícitas son mayoritariamente negativos y enfatizan sus efectos nocivos sobre la salud y el conjunto de la sociedad.

Los estudios sobre la representación de la discapacidad en la ficción televisiva, los cuales son mucho menos numerosos que los dedicados a la adicción, señalan la infrarrepresentación de estos personajes (Donaldson, 1981) y su frecuente construcción negativa (Wahl, 1978; Longmore, 1987; Barnes, 1992; Cumberbatch y Negrine, 1992) y estereotipada (Byrd, Byrd y Allen, 1977; Elliott y Byrd, 1982; Signorielli, 1989; Makas, 1993; Harnett, 2000). Wilson, Nairn, Coverdale y Panapa (1999a, 1999b) y Pirkis, Blood, Francis y McCallum (2006) observan la asociación de los personajes discapacitados con la violencia y el crimen. Diefenbach (1997) también constata una correlación frecuente entre los personajes con enfermedades psiquiátricas y el crimen violento, la cual fue corroborada una década después por Diefenbach y West (2007). Harris (2002) alerta sobre los peligros de estas falacias, teniendo en cuenta que una gran parte del imaginario social sobre las personas con algún tipo de discapacidad ha sido construido por los medios. Por último, 
un estudio reciente del Glasgow Media Group \& Time to Change (2014) denuncia la simplificación en la caracterización de los enfermos mentales y la desinformación respecto a su tratamiento médico.

La desproporción en la representación de las diferentes enfermedades ha sido objeto de un buen número de investigaciones que revelan la tendencia generalizada a eludir los problemas de salud de las personas mayores. Seale (2002) encuentra que las soap operas se inclinan más por las enfermedades graves que por las crónicas, padecidas en las representaciones por un mayor número de personajes jóvenes o de mediana edad. En esta misma línea, Cooper (2008) observa la ausencia generalizada de referencias a la menopausia en la ficción televisiva y su enfoque peyorativo en las raras ocasiones en las que se aborda el tema.

El estudio de Sharf y Freimuth (1993) sobre el tratamiento del cáncer de ovario en Thirtysomething (ABC, 1987-91) subraya el tratamiento realista de la enfermedad. El análisis de Arrington y Goodier (2004) sobre el cáncer de próstata en NYPD Blue (ABC, 1993-2005) concluye que el enfoque adoptado era poco representativo, además de simplista. El trabajo de Gray (2007) evidencia la adecuada información sobre los síntomas del cáncer de mama en The Sex and the City (HBO, 1998-2004) y su tratamiento, aunque el programa elude cuestiones tan relevantes como el impacto de la enfermedad en la vida del paciente. Mazziotti (2006) constata la reducida gama de tumores representados en las telenovelas y lo atribuye a los límites estéticos y de representación de imágenes de la ficción televisiva.

Fouts y Burggraf $(1999,2000)$ encuentran una asociación, inversamente proporcional, entre el sobrepeso de los personajes femeninos y los comentarios de los hombres al respecto, algo que no ocurre a la inversa (Fouts y Vaughan, 2002). La investigación de Greenberg, Eastin, Hofschire, Lachlan y Brownell (2003) revela que los personajes con sobrepeso suelen tener rasgos negativos (desempleado, solitario, inactividad sexual, etc.) y con frecuencia son objeto de burla por parte del resto de los personajes.

\section{Metodología}

El estudio realizado combina técnicas cuantitativas (SPSS) y aproximaciones cualitativas (socio-semiótica y script theory) para analizar la representación de los problemas de salud de una amplia muestra de personajes femeninos de la ficción televisiva española, integrada por los 84 programas de producción propia de estreno emitidos por las cadenas generalistas entre 2012 y $2013^{[1]}$. La muestra inicial comprende 709 personajes femeninos, el 15,4\% ( $\mathrm{N}=109)$ de los cuales consumen substancias tóxicas, el 11,9\% ( $=78)$ presentan diferentes grados de discapacidad (física o psíquica) y el 19,3\% ( $\mathrm{N}=137)$ padecen algún tipo de enfermedad.

La investigación se realizó en tres fases: a) visionado de los programas y creación del libro de códigos a partir de un guion de análisis socio-semiótico, construido ad hoc; b) codificación en SPSS de las variables identificadas en el guion e identificación de las storylines relacionadas con la salud de los personajes femeninos; y c) análisis descriptivo. El libro de códigos se estructura en ocho apartados: identificación del programa, salud, contexto, protagonismo, relaciones, roles, escenarios y sexualidad. Las 40 variables utilizadas en la codificación comprenden cinco variables independientes sobre salud (discapacidad, enfermedades, trastornos alimentarios, salud femenina y adicciones), y 35 variables dependientes, cinco de las cuales vehiculan datos relativos a la identificación del programa (nombre del personaje, título del programa, cadena, género de la ficción y formato). La edad de los personajes femeninos analizados se agrupó en ocho franjas, destinadas a reducir la dispersión inherente a la codificación de esta variable: 4-9; 10-14; 15-17; 18-23; 24-29; 30-45; 46-65 y más 65 años.

Como paso previo al proceso de codificación, se realizó una fase de pre-análisis destinada a detectar los posibles errores cometidos en la etapa de grabación de datos. La base de datos cuantitativa se completó con una base de datos descriptiva, construida de manera paralela al proceso de codificación en SPSS e integrada por la storyline principal sobre salud en la que participa cada personaje. El cruce de las cinco variables independientes 
con las 35 variables dependientes produjo 175 tablas de contingencia, que permitieron estructurar el análisis de los $324(45,7 \%)$ casos registrados.

\section{Resultados}

El 15,4\% (N=109) de los personajes analizados tienen algún tipo de adicción, entre las que destacan el tabaco $(8,7 \% ; \mathrm{N}=62)$, el alcohol $(7,6 \% ; \mathrm{N}=54)$ y las drogas $(2,8 \% ; \mathrm{N}=20)$. Los personajes femeninos que presentan algún tipo de trastorno psíquico, enfermedad mental o discapacidad física constituyen el 11,9\% ( $\mathrm{N}=78)$ de la muestra ${ }^{[2]}$. Los trastornos de carácter psíquico son los más frecuentes $(5,2 \% ; \mathrm{N}=37)$, seguidos por la discapacidad física ( $4,9 \% ; \mathrm{N}=35)$ y las enfermedades mentales $(2,8 \% ; \mathrm{N}=20)$. Por último, el 19,3\% $(\mathrm{N}=137)$ de los personajes tienen problemas de salud del cuerpo: graves o leves $(7,0 \% ; \mathrm{N}=50$ en cada caso), relacionados con la edad $(3,6 \% ; \mathrm{N}=26)$, de carácter crónico $(1,6 \% ; \mathrm{N}=12)$, trastornos alimentarios $(0,9 \% ; \mathrm{N}=7)$ y una miscelánea $(3,1 \% ; \mathrm{N}=22)$ integrada por representaciones esporádicas, como, por ejemplo, las complicaciones en los embarazos o los abortos espontáneos.

Los personajes femeninos de 30 a 45 años consumen más alcohol $(11,2 \% ; \mathrm{N}=29)$, tabaco $(14,0 \% ; \mathrm{N}=36)$ y drogas $(4,2 \% ; \mathrm{N}=11)$ que el resto, en consonancia con su elevada representación (36,2\%; $\mathrm{N}=257)$. El segundo grupo en el consumo de alcohol está integrado por las jóvenes de 15 y $17(10,3 \% ; \mathrm{N}=3)$, seguidas por las mujeres de 46 a 65 años (8,0\%; $\mathrm{N}=11)$.

La mayor parte de las fumadoras habituales, la adicción más frecuente en la ficción doméstica, tienen entre 30 y 45 años (14,0\%; $N=36)$, seguidas por el grupo de 46 a 65 (9,6\%; $N=13)$. Por una parte, el consumo de tabaco se presenta, generalmente, como un hábito normalizado, aunque la ficción asume una cierta función didáctica al incluir personajes que lamentan los perjuicios del consumo de tabaco y los efectos del humo en entornos cerrados o en presencia de menores. Por otra, la vinculación de las fumadoras más recalcitrantes con personajes negativos homologa la disforia que suscitan dichos personajes con esta adicción.

Las limitaciones físicas afectan a un reducido número de personajes femeninos de todas las franjas de edad, mientras que los trastornos psíquicos son más frecuentes entre las jóvenes de 15 y 29 años. En general, la ficción no relaciona de manera drástica la discapacidad física con la falta de atractivo, pues el 51,4\% $(\mathrm{N}=18)$ de las mujeres con algún tipo de limitación de este tipo son consideradas atractivas por su entorno.

La discapacidad física de la mayor parte de los 35 personajes femeninos $(4,9 \%)$ que la padecen afecta a la movilidad, la visión o la audición. Pese a que la pérdida de movilidad siempre es el resultado de episodios dramáticos y que a las mujeres afectadas les cuesta mucho aceptar sus secuelas, se caracterizan generalmente por su afán de superación. El mayor número de disfunciones visuales de los personajes femeninos (sobre todo entre los 18 y 29 años) respecto de las auditivas (generalmente mayores de 65 años) resulta funcional al guion por la autonomía comunicativa de las primeras. Además, al igual que ocurre con los problemas de movilidad causados por algún accidente, la ficción tiende a ofrecer una perspectiva optimista de este tipo de discapacidad mediante ejemplos de recuperación de la visión por intervención de la cirugía. A diferencia del drama, el tratamiento de la discapacidad física en la comedia (10 personajes sobre un total de 35) suele resultar controvertido por la labilidad de la frontera que separa la ironía del cinismo en la representación de algunas mujeres con problemas auditivos, que intencionadamente malinterpretan lo que oyen, o que no reconocen sus limitaciones de movilidad debido a su sobrepeso.

Los trastornos psíquicos suelen girar en torno a las relaciones sentimentales y familiares, particularmente a la violencia de género, que con frecuencia aboca a las víctimas a buscar la muerte (suicidio o intento de suicido) o a provocarla (asesinato). El fallecimiento de la pareja y los problemas sentimentales constituyen dos de los principales desencadenantes de la depresión, e incluso de la locura, mientras que otros trastornos de este tipo son justificados narrativamente a partir de una tragedia o disfunción familiar (el fallecimiento de un hijo, su vinculación con las drogas o la mafia, la desintegración de la familia, los problemas económicos, etc.). 
El amplio abanico de enfermedades y trastornos físicos afecta principalmente a los personajes femeninos de 15 a 17 años $(37,9 \% ; \mathrm{N}=11)$ y a las mujeres de más de $65(47,4 \% ; \mathrm{N}=18)$. En el primer caso, se trata del grupo que padece un mayor número de enfermedades graves, leves, crónicas y trastornos alimentarios, mientras que el segundo grupo está aquejado principalmente de enfermedades relacionadas con la edad. Los problemas de salud leves complementan historias que se abren y se cierran en una misma entrega, mientras que los problemas de salud graves permiten desarrollar storylines con un recorrido narrativo más largo. Además de la sintomatología y las consecuencias, la gravedad de una enfermedad también está condicionada por la localización temporal de la ficción televisiva. Por ejemplo, las complicaciones del parto o del postparto son relativamente frecuentes en las historias ambientadas en el pasado, al igual que algunas enfermedades erradicadas en España en la actualidad (como la poliomielitis o la lepra) y un amplio catálogo de intoxicaciones y envenenamientos. La representación de las enfermedades crónicas es meramente testimonial y comprende tanto las afecciones graves (problemas cardiológicos), como otras enfermedades mucho más leves (alergia a los lácteos, hipersensibilidad a productos de limpieza, etc.). Las dolencias asociadas con la edad comienzan a manifestarse en el grupo de mujeres de 46 a 65 años $(5,1 \% ; \mathrm{N}=7)$, a las que cabe añadir seis personajes más jóvenes de las ficciones de época. No obstante, este tipo de trastornos afecta particularmente a los personajes femeninos mayores de 65 años $(34,2 \%$; $N=13)$, quienes padecen artrosis, trastornos oculares y alteraciones de la presión arterial.

Finalmente, cabe señalar que los trastornos alimentarios son abordados de manera muy esporádica, al igual que las enfermedades graves con mayor incidencia en las mujeres, como, por ejemplo, el cáncer de mama. La representación del ciclo fértil de la mujer introduce ocasionalmente la menopausia, pero siempre en clave de humor en la comedia.

\section{Discusión y conclusiones}

El análisis realizado pone de relieve la ambivalencia de las representaciones sobre los trastornos de salud en la ficción televisiva española, que se debate entre la recurrencia de estereotipos arraigados en los diferentes géneros y formatos, y la vocación didáctica de buena parte de los relatos. En general, se observa una cierta despreocupación por la promoción de hábitos saludables en esferas tan relevantes como la alimentación, el ejercicio físico o la sexualidad, constada en otras investigaciones (Seale, 2002), a lo que cabría añadir la omisión generalizada de las consecuencias del consumo de alcohol y de tabaco. Además, a pesar del número relativamente elevado de personajes que padecen algún tipo de enfermedad, este tipo de representaciones suelen resultar determinantes únicamente en la caracterización de algunos personajes secundarios o que tan sólo intervienen esporádicamente en los relatos. Otro aspecto destacable es el desinterés generalizado por la salud femenina.

La ambivalencia es aún más clara en la representación de adicciones y malos hábitos relacionados con el alcohol y el tabaco. A diferencia del consumo de alcohol de las mujeres españolas, inversamente proporcional a su edad según los datos de las últimas encuestas de salud en España realizadas por el Ministerio de Sanidad, Servicios Sociales e Igualdad [Msssi] y el Instituto Nacional de Estadística [INE] (2013), y el Programa de Encuestas sobre Alcohol y Drogas en España [Edades] (2014) ${ }^{[3]}$, la ficción televisiva lo asocia sobre todo con los personajes femeninos de 30 a 45 años. Se produce, por consiguiente, una cierta disociación entre el consumo de alcohol y la juventud, pero raramente se abordan sus efectos y en general se asocia con la diversión. El consumo de tabaco tampoco refleja la correspondencia entre la sociedad española y la ficción de producción propia; pues, mientras que el grupo de personajes de 30 a 45 años incluye un mayor número de fumadoras que el resto de las franjas de edad (al igual que ocurría con el consumo de alcohol), el de tabaco es semejante entre las mujeres españolas de 25 a 45 años (INE, 2013; Edades, 2014). Se trata de la adicción más recurrente en el análisis realizado, cuyo incremento en las representaciones es paulatino pero constante (McGee y Ketchel, 
2006), y, aun así, generalmente carece de relevancia narrativa (Sone, 1999). Como ocurría con el consumo de alcohol, sus consecuencias se eluden sistemáticamente (Cumberbatch y Gauntlett, 2005; McGee y Ketchel, 2006), si bien se registra un mayor número de situaciones en las que se reconoce un cierto interés por exponer los efectos nocivos del tabaco mediante la caracterización negativa de las fumadoras. El consumo de drogas, en cambio, siempre es representado de forma negativa (Christensen et al., 2000; Cumberbatch y Gauntlett, 2005; McGee y Ketchel, 2006).

La representación de la discapacidad y de la enfermedad también refleja la diferencia entre la realidad y la ficción televisiva española, sobre todo en lo referente a las mujeres mayores. Así, mientras que los datos más recientes del INE (2008) sitúan en el 17\% la dependencia de las mujeres de 65 a 69 años, un porcentaje que se incrementa en progresión geométrica hasta llegar al 78,3\% de las mayores de 90 años, únicamente el 14,3\% (N $=5$ ) de los personajes mayores de 65 años padecen algún condicionante relacionado con el envejecimiento. Se trata de un dato coherente con una sobrerrepresentación de la juventud y la belleza que elude sistemáticamente a las mujeres mayores y, por consiguiente, los trastornos asociados con la edad (Seale, 2002).

El culto a la juventud y la belleza incide, asimismo, en el elevado número de trastornos mentales (psicológicos y psiquiátricos) respecto de los trastornos físicos que padecen los personajes femeninos de 15 y 29 años. Sin embargo, a diferencia de las adicciones, los resultados del presente estudio distancian las representaciones de la discapacidad y los trastornos mentales de otras investigaciones sobre ficción norteamericana (Pirkis et al., 2006; Diefenbach y West, 2007), pues los personajes femeninos de este grupo no suelen ser mujeres negativas, ni tampoco agresivas o inestables. Se observa, en cambio, una tendencia a la simplificación de este tipo de personajes, caracterizados casi exclusivamente en torno a su discapacidad (Harnett, 2000; Makas, 1993). Ocasionalmente, la introducción de la discapacidad física ofrece un desenlace positivo (recuperación de la vista o de la movilidad) destinado a satisfacer el deseo de conclusiones felices de la ficción, una tendencia generalizada de indudable transcendencia considerando la mayor eficacia los mensajes relacionados con la salud en la ficción respecto de otros géneros televisivos (Murphy et al., 2013).

El análisis realizado también avala la premisa de Seale (2002) sobre la preferencia de la ficción por las enfermedades graves, abordadas como un evento específico en un arco narrativo de corta o media duración respecto de las enfermedades crónicas. Al igual que ocurría con la discapacidad, la representación de estas enfermedades se decanta por las historias con final feliz.

La investigación representa una aportación novedosa a este campo de estudio mediante la implementación de una metodología destinada a abordar el análisis de muestras de tamaño medio, que combina la codificación en SPSS de las variables identificadas a partir de un guion socio-semiótico con el análisis descriptivo de los 324 casos registrados. Además de la versatilidad de la metodología, que puede ser adaptada a otros géneros y formatos televisivos, los resultados obtenidos podrían ser complementados con otros análisis de la recepción para poder determinar, de la manera más precisa posible, el impacto de la ficción televisiva española en la comunicación sobre salud.

\section{Agradecimientos}

El artículo se enmarca en el proyecto "La construcción social de la mujer en la ficción televisiva y la Web 2.0: estereotipos, recepción y retroalimentación” (FEM2012-33411), financiado por el Ministerio de Economía y Competitividad. En esta parte de la investigación han participado, además de las autoras, Deborah Castro, Mariluz Sánchez, Belén Granda, Tatiana Hidalgo, Elsa Soro y Karina Tiznado (investigadoras) y Marc Bellmunt, Germán Muñoz, Lucía Trabajo, Estitxu Garay y Amaia Neracan (colaboradores). 


\section{Referencias}

Arrington, M. I., y Goodier, B. C. (2004). Prostration before the law: Representations of illness, interaction, and intimacy in the NYPD Blue prostate cancer narrative. Popular Communication, 2(2), 67-84. doi: 10.1207/ s15405710pc0202_1

Barnes, C. (1992). Disabling Imagery and the Media. Halifax: Ryburn/British Council of Disabled People (BCODP). Recuperado de http://disability-studies.leeds.ac.uk/files/library/Barnes-disabling-imagery.pdf

Bouman, M. P. (2014). Entertainment-Education in Western Countries. En T. L. Thonmpson (ed.), Encyclopedia of Health Communication (pp. 1-3). Londres, Reino Unido: SAGE. Recuperado de http://www.media-gezondheid.nl/beheer/data/cmg.desh26.nl/uploads/Publicaties_en_downloads/Health _Encyclopedia_EE_in_western_settings_Martine_Bouman.pdf

Bouman, M. P., Maas, L., y Kok, G. (1998). Health education in television entertainment - Medisch Centrum West: a Dutch drama serial. Health Education Research, 13(4), 503-18.

Breed, W., De Foe, J. R., y Wallack, L. (1984). Drinking in the mass media: a nine year project. Journal of Drug Issues, 14(4), 655-664. doi: 10.1177/002204268401400405

Breed, W., y De Foe, J. (1981). The portrayal of the drinking process on prime-time television. Journal of Communication, 31(1), 58-67. doi: 10.1111/j.1460-2466.1981.tb01205.x

Bury, M. (2001). Illness narratives: fact or fiction? Sociology of Health \& Ilness, 23(3), 263-285.

Byrd, E. K., Byrd, P. D., y Allen, C. M. (1977). Television programming and disability.Journal of Applied Rehabilitation Counseling, 8(1), 28-32.

Chang, J. E., y Roth, W. E. (2000). When Is Cranberry Sauce Shaped Like a Can? An Investigation of Cultural Capital, Gender and Consumption in Television Programming. En C. Otnes (ed.), Gender and Consumer Behavior, Volume 5 (pp. 107-124). Urbana, IL, Estados Unidos: Association for Consumer Research.

Christensen, P. G., Henriksen, L., y Roberts, D. F. (2000). Substance Use in Popular Prime-Time Television. Washington, DC: Office of National Drug Control Policy.

Cooper, R. (2008). Prime Time; TV menopause, queerly a case for review. SQS. Journal of Queer Studies in Finland, 3(2), 30-37.

Cruz, J., y Wallack, L. (1986). Trends in tobacco use on television. American Journal of Public Health, 76(6), 698-699. doi: 10.2105/AJPH.76.6.698

Cullen, J., Sokol, N. A., Slawek, D., Allen, J. A., Vallone, D., y Healton, C. (2011). Depictions of tobacco use in 2007 broadcast television programming popular among US youth. Archives of pediatrics \& adolescent medicine, 165(2), 147-51. doi:10.1001/archpediatrics.2010.276

Cumberbatch, G., y Gauntlett, S. (2005). Smoking, alcohol, and drugs on television: a content analysis. London: Ofcom. Recuperado de http://stakeholders.ofcom.org.uk/binaries/research/radio-research/smoking.pdf

Cumberbatch, G., y Negrine, R. (1992). Images of Disability on Television. London: Routledge.

Diefenbach, D. L. (1997). The portrayal of mental illness on prime-time television. Journal of Community Psychology, 25(3), 289-302. doi: 10.1002/(SICI)1520-6629(199705)25:33.0.CO;2-R

Diefenbach, D. L., y West, M. D. (2007). Television and attitudes toward mental health issues: Cultivation analysis and the third-person effect. Journal of Community Psychology, 35(2), 181-195. doi: 10.1002/jcop.20142

Donaldson, J. (1981). The visibility and image of handicapped people on televisión. Exceptional Children, 47(6), 41317. doi: $10.1177 / 001440298104700602$

Elliott, T. R., y Byrd, E. K. (1982). Media and disability. Rebabilitation Literature, 43(11-12), 348-355.

Eslick, G. D., y Eslick, M. G. (2009). Smoking and The Simpsons. The Medical Journal of Australia, 190(11), 637-639.

Fouts, G., y Burggraf, K. (1999). Television situation comedies: female body images and verbal reinforcements. Sex Roles, 40(5), 473-481. doi: 10.1023/A:1018875711082

Fouts, G., y Burggraf, K. (2000). Television situation comedies: Female weight, male negative comments, and audience reactions. Sex Roles, 42(9), 925-932. doi: 10.1023/A:1007054618340 
Fouts, G., y Vaughan, K. (2002). Television situation comedies: Male weight, negative references, and audience reactions. Sex Roles, 46(11), 439-442. doi: 10.1023/A:1020469715532

Fuenzalida, V. (1992). Telenovelas y desarrollo. Diálogos de la comunicación, 33, 35-40.

Fuenzalida, V. (1996). La apropiación educativa de la telenovela. Diálogos de la comunicación, 44, 91-105.

Fuenzalida, V. (2011). Resignificación de la educación televisiva: desde la escuela a la vida cotidiana. Una visión desde América Latina. Comunicar, 36, 15-23.

Furnham, A., Ingle, H., Gunter, B., y McClelland, A. (1997). A content analysis of alcohol portrayal and drinking in British television soap operas. Health Eduction Research, 12(4), 519-29. doi: 10.1093/her/12.4.519

Glasgow Media Group \& Time to Change (2014). Making a Drama out of a Crisis: Authentic Portrayals of Mental Illness in TV Drama. Recuperado de http://www.time-to-change.org.uk/sites/default/files/Making_a_drama_ out_of_a_crisis.pdf

Glick, D., Berkanovic, E., Stone, K., Ibarra, L., Jones, M. C., Rosen, B., ... Richardes, D. (1998). Health Education goes Hollywood: Working with prime-time and daytime entertainment television for immunization promotion. Journal of Health Communication, 3(3), 263-282. doi: 10.1080/108107398127364

Gray, J. B. (2007). Interpersonal Communication and the Illness Experience in the Sex and the City Breast Cancer Narrative. Communication Quarterly, 55(4), 397-414. doi: 10.1080/01463370701657889

Greenberg, B S. (1981). Smoking, drugging, and drinking in top rated TV series. Journal of Drug Education, 11(3), 227-234. doi: 10.2190/DJ5P-KQW5-HPJC-QURL

Greenberg, B. S., Eastin, M., Hofschire, L., Lachlan, K., y Brownell, K. D. (2003). Portrayals of Overweight and Obese Individuals on Commercial Television. American Journal of Public Health, 93(8), 1342-1348.

Hanewinkel, R., y Wiborg, G. (2008). Smoking in a popular German television crime series 1985-2004. Preventive medicine, 46(6), 596-8. doi: 10.1016/j.ypmed.2008.02.012

Hanneman, G., y McEwen, W. (1976). The use and abuse of drugs: An analysis of mass media content. En R. E. Ostman (ed.), Communication Research and Drug Education (pp. 65-88). Beverly Hills, CA, Estados Unidos: Sage.

Hansen, A. (1986). The portrayal of alcohol on television. Health Education Journal, 45(3), 127-131. https://doi.or $\mathrm{g} / 10.1177 / 001789698604500302$

Harnett, A. (2000). Escaping the "evil avenger" and the "supercrip": images of disability in popular television. Irish Communications Review, 8, 21-29.

Harris, L. (2002). Disabled sex and the movies. Disability Studies Quarterly, 22(4), 144-162. doi: http:// dx.doi.org/10.18061/dsq.v22i4.378

Hayes, M., Ross, I. E., Gasher, M., Gutstein, D., Dunn, J. R., y Hackett, R. A. (2007). Telling stories: news media, health literacy and public policy in Canada. Social Science and Medicine, 64, 1842-1852. doi:10.1016/ j.socscimed.2007.01.015

Hazan, A. R., y Glantz, S. A. (1995). Current trends in tobacco use on prime-time fictional television. American Journal of Public Health, 85(1), 116-117. doi: 10.2105/AJPH.85.1.116

Hooker, C., y Pols, H. (2006). Health, Medicine, and the Media. Health and History, 8(2), 1-13. doi: $10.2307 / 40111540$.

Hundley, H. L. (1995). The Naturalization of Beer in Cheers. Journal of Broadcast and Electronic Media, 39(3), 350359. doi:10.1080/08838159509364311

Igartúa, J. J., y Casanova, J. V. (2016). Identification With Characters, Elaboration, and Counterarguing in Entertainment-Education Interventions Through Audiovisual Fiction. Journal of Health Communication, 21(3), 293-300. doi: 10.1080/10810730.2015.1064494

Instituto Nacional de Estadística (INE). (2008). Encuesta sobre discapacidades, autonomía personal y situaciones de dependencia. Recuperado de http://www.ine.es/dyngs/INEbase/es/operacion.htm?c=Estadistica_C\&cid=125 $4736176782 \&$ menu $=$ resultados\&idp $=1254735573175$ 
Instituto Nacional de Estadística (INE). (2013). Encuesta Nacional de Salud 2011 - 2012. Recuperado de http://www.ine.es/dyngs/INEbase/es/operacion.htm?c=Estadistica_C\&cid=1254736176783\&menu=res ultados\&idp $=1254735573175$

Kaiser Family Foundation. (2004). Entertainment Education and Health in the United States. Recuperado de https://kaiserfamilyfoundation.files.wordpress.com/2013/01/entertainment-education-and-health-in-theunited-states-issue-brief.pdf.

Kanda, H., Okamura, T., Turin, T. C., Hayakawa, T., Kadowaki, T., y Ueshima, H. (2006). Smoking scenes in popular Japanese serial television dramas: descriptive analysis during the same 3 -month period in two consecutive years. Health promotion international, 21(2), 98-103. doi: 10.1093/heapro/dal007

Lantz P. M., y Booth, K. M. (1998). The social construction of the breast cancer epidemic. Social Science and Medicine, 46(7), 907-918.

Longmore, P. K. (1987). Screening stereotypes: images of disabled people in television and motion pictures. En A. Gartner y T. Joe (eds.), Images of Disabled, Disabling Images (pp. 65-78). Nueva York, Estados Unidos: Praeger.

Lyons, A., McNeill, A., y Britton, J. (2013). Tobacco imagery on prime time UK television. Tobacco Control. doi:10.1136/ tobaccocontrol-2012-050650

Makas, E. (1993). Changing channels: the portrayal of people with disabilities on television. En G. L. Berry y J. K. Asamen (eds.), Children and Television (pp. 255- 68). Newbury Park, CA, Estado Unidos: Sage.

Makoul, G., y Peer, L. (2004). Dissecting the doctor shows: A content analysis of ER and Chicago Hope. En L. D. Friedman (ed.), Cultural sutures. Medicine and media (pp. 244--260). Durham, NC, Estados Unidos: Duke University Press.

Mathios, A., Avery, R., Bisogni, C., y Shanahan, J. (1998). Alcohol Portrayal on Prime-Time Television: Manifest and Latent Messages. Journal of Studies on Alcohol, 59(3), 305-310. doi: http://dx.doi.org/10.15288/ jsa.1998.59.305

Mazziotti, N. (2006). Telenovela y prácticas sociales. Bogotá: Norma.

McEwen, W. J., y Hanneman, G J. (1974). The depiction of drug use in television programming. Journal of Drug Education, 4(3), 281-293. doi: 10.2190/45GQ-RQ05-53AG-GEPY

McGee, R., y Ketchel, J. (2006). Tobacco imagery on New Zealand television 2002-2004. Tobacco Control, 15(5), 412-414. doi: 10.1136/tc.2006.016048

Ministerio de Sanidad, Servicios Sociales e Igualdad (Msssi) e Instituto Nacional de Estadística (INE). (2013). Encuesta Nacional de Salud. España 2011/12. Serie Informes monográficos no 1. Consumo de alcohol. Madrid. Recuperado de http://www.msssi.gob.es/estadEstudios/estadisticas/encuestaNacional/encuestaNac2011/informesMonog raficos/ENSE2011_12_MONOGRAFICO_1_ALCOHOL4.pdf

Murphy, S. T., Frank, L. F., Chatterjee, J. S., y Baezconde-Garbanati, L. (2013). Narrative versus nonnarrative: The role of identification, transportation, and emotion in reducing health disparities. Journal of Communication, 63(1), 116-137. doi: $10.1111=$ jcom. 12007

Parikh, N. (2007). Medicine and media: a symbiotic relationship? Virtual Mentor. American Medical Association Journal of Ethics, 9(3), 163-165.

Parsons, S., Rissel, C., y Douglas, W. (1999). Alcohol Portrayal in Australian Prime Time Television in 1990 and 1997. Australian and New Zealand Journal of Public Health, 23(1), 67-71. doi: 10.1111/ j.1467-842X.1999.tb01207.x.

Payton, F. C., Kvasny, L., y Kiwanuka-Tondo, J. (2014). Online HIV prevention information: How black female college students are seeking and perceiving. Internet Research: Electronic Networking Applications and Policy, 24(4), 520-542.

Pintor-Holguín, E., Rubio-Alonso, M., Herreros Ruiz-Valdepen\#as, B., Corral-Pazos de Provens, O. J., Buzo\#n-Rueda, L., y Vivas-Rojo, E. (2012). Series médicas en televisión vistas por estudiantes de medicina. Educación Médica, 15(3), 161-166.

Pirkis, J., Blood, W., Francis, C., y McCallum, K. (2006). On-screen portrayals of mental illness: Extent, nature, and impacts. Journal of Health Communication, 11(5), 523- 541. doi: 10.1080/10810730600755889 
Powers, A., y Andsager, J. L. (1999). How newspapers farmed breast implants in the 1990's. Journalism \& Mass Communication Quarterly, 76(3), 551-564.

Programa de Encuestas sobre Alcohol y Drogas en España (Edades). (2014). Consumo, percepciones y opiniones ante las drogas. Recuperado de http://www.pnsd.msssi.gob.es/profesionales/sistemasInformacion/sistemaInformacion /pdf/2015_Informe_EDADES.pdf

Razum, O., Weishaar H., y Schaeffer D. (2016). Health Literacy: strengthening agency for changing structures? International Journal of Public Health, 4, 153-189. doi: 10.1007/s00038-016-0788-x

Russell, C. A., Russell, D. W., y Grube, J. W. (2009). Nature and Impact of Alcohol Messages in a Youth-Oriented Television Series. Journal of Advertising, 38(3), 97-112. doi: 10.2753/JOA0091-3367380307

Russell, C. A., y Russell, D. W. (2009). Alcohol Messages in Prime-Time Television Series. The Journal of Consumer Affairs, 43(1), 108-128. doi: 10.1111/j.1745-6606.2008.01129.x

Seale, C. (2002). Media \& Health. London: Sage.

Sharf, B. F., y Freimuth, V. S. (1993). The construction of illness on entertainment television: Coping with cancer on thirtysomething. Health Communication, 5(3), 141-160. doi:10.1207/s15327027hc0503_1

Signorielli, N. (1989). The stigma of mental illness on televisión. Journal of Broadcasting \& Electronic Media, 33(3), 325-331. doi:10.1080/08838158909364085

Singhal, A., Cody, M., Rogers, E., y Sabido, M. (eds.). (2004). Entertainment-education and Social Change: History, Research, and Practice. Mahwah, NJ: Lawrence Erlbaum.

Singhal, A., y Rogers, E. M. (1999). Entertainment\#education. A Communication strategy for social change. Mahwah, NJ: Lawrence Erlbaum.

Singhal, A., y Rogers, E. M. (2004). The Status of Entertainment-Education Worldwide. En A. Singhal, M. Cody, E. Rogers y M. Sabido (eds.), Entertainment Education and social change: History, research and practice (pp. 3-20). Mahwah, NJ, Estados Unidos: Lawrence Erlbaum.

Sone, T. (1999). Tobacco-related scenes in television dramas for young Japanese audiences. Tobacco Control, 8(3), 350.

Tufte, T. (2004). Eduentretenimiento en la comunicación para el vih/sida más allá del mercadeo, hacia el empoderamiento, Investigación \& Desarrollo, 12(1), 24-43.

Valente, T. W., Murphy, S., Huang, G., Gusek, J., Greene, J., y Beck, V. (2007). Evaluating a minor storyline on ER about teen obesity, hypertension, and 5 A day. Journal of Health Communication, 12(6), 551-566. doi: 10.1080/10810730701508385.

Van Hoof, J. J., De Jong, M. D., Fennis, B. M., y Gosselt, J. F. (2009). There's alcohol in my soap: portrayal and effects of alcohol use in a popular television series. Health Education Research, 24(3), 421 - 29. doi: 10.1093/her/cyn037

Verma, T., Adams, J., y White, M. (2007). Portrayal of healt-related behaviours in popular UK television soap operas. Journal of Epidemiology and Community Health, 61(7), 575-577. doi: 10.1136/jech.2006.050757

Wahl, O. (1978). Mental illness in the media: An unhealthy condition. Disabled USA, 2, 23-24.

Weaver, R., Wilson, I., y Langendyk, V. (2014). Medical professionalism on television: student perceptions and pedagogical implications. Health, 18(6), 597-612. doi: 10.1177/1363459314524804

Wilkin, H. A., Valente, T. W., Murphy, S., Cody, M. J., Huang, G., y Beck, V. (2007). Does Entertainment-Education work with latinos in the United States? Identification and the effects of a telenovela breast cancer storyline. Journal of Health Communication, 12(5), 455-469. doi: 10.1080/10810730701438690.

Wilson, C., Nairn, R., Coverdale, J., y Panapa, A. (1999a). Constructing mental illness as dangerous: a pilot study. Australian and New Zealand Journal of Psychiatry, 33(2), 240-247. doi: 10.1046/j.1440-1614.1999.00542.x

Wilson, C., Nairn, R., Coverdale, J., y Panapa, A. (1999b). Mental illness depictions in prime-time drama: Identifying the discursive resources. Australian and New Zealand Journal of Psychiatry, 33(2), 232-239. doi: 10.1046/ j.1440-1614.1999.00543.x 


\section{Notas}

[1] Se han excluido únicamente los personajes secundarios ocasionales que figuran en menos de tres episodios.

[2] Algunos personajes tienen más de una adicción, al igual que ocurre con la discapacidad y la enfermedad.

[3] El 57,2\% afirma beber habitualmente (mínimo una vez por semana) o de manera ocasional (INE, 2013), mientras que el $27,1 \%$ de la población mayor de 15 años declara fumar a diario o de manera ocasional.

* $\quad$ Artículo de investigación

\section{Licencia Creative Commons CC BY 4.0}

Cómo citar este artículo: Lacalle, C., y Gómez Morales, B. (2018). Educar entreteniendo: los problemas de salud de los personajes femeninos en la ficción TV española. Signo y Pensamiento, 37(73). https://doi.org/ 10.11144/Javeriana.syp37-73.eeps 